\title{
Gestión estratégica del riesgo y su importancia en las buenas prácticas empresariales
}

\author{
Fecha de recepción: 01-12-2020 • Fecha de aceptación: 25-01-2021 • Fecha de publicación: 10-02-2021
}

Ricardo Bautista Penna ${ }^{1}$

Federación Patronal Seguros S.A., Argentina

ricardo.penna@econo.unlp.edu.ar

https://orcid.org/0000-0001-7659-1423

Fernando Gustavo Pérez Sisa²

Universidad Central del Ecuador fjperez@uce.edu.ec https://orcid.org/0000-0002-6279-4366

\section{RESUMEN:}

La falta de gestión profesional en el tratamiento de los diferentes riesgos que enfrentan las organizaciones genera diversas consecuencias positivas o negativas según el escenario empresarial en el que se presente, mediante un análisis documental enfocado en aquellos riesgos dónde no es posible valerse de herramientas matemáticas o estadísticas para poder sobrellevar específicamente una determinada actividad o proyecto, se plantea un sistema que aporte ya sea a la gerencia interna o asesoría externa, la facilidad de implementar con una mayor preponderancia en la estructura de cada entidad las política de riesgos estableciendo un marco referencial, objetivos, alcances, definiciones, funciones y responsabilidades en las diferentes estructuras que puedan tener las organizaciones siendo vital una buena práctica administrativa para transparentar todos los procesos e información para la toma de decisiones. Los administradores necesariamente deben combinar sus capacidades y experiencias dentro de las empresas para afrontar los diversos riesgos o eventualidades que se puedan ir presentado en la institución, de esta forma se podrá reducir o mitigar el impacto que este pueda tener. 


\section{ABSTRACT}

The lack of professional management in the treatment of the different risks faced by organizations generates various positive or negative consequences depending on the business scenario in which it occurs, through a documentary analysis focused on those risks where it is not possible to use mathematical or statistical tools to specifically cope with a particular activity or project, a system is proposed that provides either internal management or external advice, the facility to implement with a greater preponderance in the structure of each entity the risk policy establishing a reference framework, objectives, scope, definitions, roles and responsibilities in the different structures that organizations may have, being vital a good administrative practice to make transparent all processes and information for decision making. Managers must necessarily combine their capabilities and experiences within the companies to face the various risks or eventualities that may arise in the institution, thus reducing or mitigating the impact that this may have.

\section{KEYWORDS: risk management, practices, assessment, management.}




\section{Introducción}

El riesgo representa un escenario de incertidumbre empresarial, generando varias complicaciones en la organización debido al mal manejo administrativo y prácticas que los directivos ejecuten, en base a eso, es necesario establecer una gestión adecuada, centrándose en las estrategias de la compañía, mismas que permitirán en muchos escenarios reducir el posible impacto positivo o negativo que la empresa pueda enfrentar. El objetivo de la investigación se centra en brindar herramientas claras y oportunas para tratar el riesgo y su impacto en las prácticas empresariales.

Los riesgos impactan en todas las actividades que desarrolla un administrador en la dirección estratégica, producción, recursos humanos, planeación financiera, marketing; ninguna función del management resulta inmune a las inclemencias de los riesgos y sin embargo, son pocas las áreas que desde sus programas o planificación formal consideran con profesionalismo estas eventualidades que atentan contra el logro de sus objetivos (Aristizábal, 2019).

Es importante advertir que los riesgos que se referenciarán en este artículo no se corresponden con los riesgos financieros, comerciales o especulativas, en los que se consideran las variaciones sobre ganar más o ganar menos, sino que se dará tratamiento a los riesgos puros, es decir, a aquellos en los que por la naturaleza de sus efectos pueden implicar pérdidas reales del patrimonio y recursos (Aristizábal, 2019).

Estos se encuentran presentes en absolutamente todas las actividades de los agentes económicos, pero obviamente no atañen solo a administradores y empresarios. Si bien el concepto de riesgo es relativamente reciente, el mismo acompaña a la humanidad desde sus inicios y forma parte de su naturaleza, siendo el ser humano el único de los seres vivos que es consciente del riesgo de su propia muerte. Por ello, nuestros antepasados se arriesgaban cada vez que debían abandonar la seguridad de las cuevas y refugios para conseguir alimento (Aristizábal, 2019).

Más allá de su incipiente consideración en la matemática indo-árabe, la conciencia del riesgo como concepto y más aún su tratamiento específico en las ciencias de la administración, resultan relativamente recientes. En la actualidad, puede resultarnos difícil entender el pensamiento de nuestros predecesores dónde se desconocía el sentido moderno de esta noción, dónde el futuro esperable es el resultado de algo más que el antojo de los dioses (o el espejo del pasado) y que los hombres y mujeres no son pasivos ante la naturaleza (Noa, 2017).

El riesgo hace mención a la posibilidad de que ocurra un acontecimiento incierto, fortuito y de consecuencias económicas negativas o dañosas (esto último es lo que diferencia al riesgo desde la expectativa). A su vez, no debe confundirse la noción del riesgo con la de amenaza ya que, si bien ambos son contrarios a la voluntad del interviniente, en el primero existe una decisión previa de enfrentarlo para sobrellevar una determinada situación u obtener un beneficio, mientras que la amenaza resulta ajena a toda elección u acción que pueda llevar a cabo quién la padece. En este sentido, se establece que el riesgo mismo dependerá de la existencia simultánea tanto del futuro, como de las variables aleatorias (Noa, 2017).

De acuerdo con el autor Carvajal (2019), este se ejecuta en función de dos variables: amenaza 
y vulnerabilidad. Ambas son condiciones necesarias para expresar riesgo, y el riesgo se expresa como la probabilidad de pérdida en una ubicación geográfica definida y en un momento específico. Aunque los eventos naturales no siempre son controlables, la vulnerabilidad sí lo es.

Resulta necesario señalar que la gestión de riesgos también tiene un lado oscuro y es el que está relacionado al ingrato rol del mensajero que porta malas noticias como el de alertar o informar sobre un riesgo determinado. Pretender que los riesgos no se van a presentar porque no los identifiquemos, equivale a afirmar que las cosas no se caían antes que Newton formulara la Ley de la gravedad. Lejos de esta postura, la detección de un riesgo debe ser reconocida como una gran fortuna, ya que la posibilidad del evento dañoso siempre estuvo, la diferencia es que al reconocerlo se ha transformado la imprevisión en un riesgo identificado, el cuál si se lo trata adecuadamente puede ser gestionado con éxito y así eliminar o reducir la pérdida potencial (Carvajal, 2019).

\subsection{Riesgos objetivos y específicos}

Al analizar los riesgos para las empresas se afirma que la primera parte de la definición de riesgo es la incertidumbre, es decir, que resulta imposible predecir el resultado de una determinada situación en un momento dado y que esta imposibilidad se debe principalmente al desconocimiento respecto al futuro y, por el contrario, si sabe con certeza lo que sucederá, no hay riesgo (Noa, 2017).

En un sentido estricto se puede diferenciar dos estadios principales que se encuentran entre los extremos de ocurrencia de los eventos como lo son las situaciones que se presentan en certeza total o en la ignorancia absoluta, que se denominan riesgo e incertidumbre (Figura 1), dependiendo si se conocen la existencia de probabilidades matemáticas de ocurrencia o solo se puede inferir una probabilidad subjetiva, respectivamente (Noa, 2017).

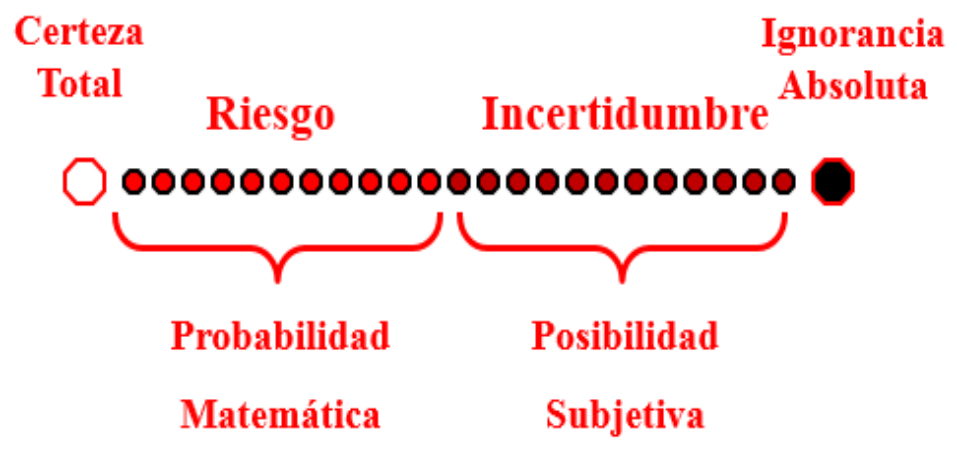

Figura 1. Probabilidad subjetiva del riesgo

Fuente: elaboración propia

A partir de dicha diferencia (Figura 2) es que se puede discriminar a los riesgos por su sistema de valoración entre los riesgos objetivos y los específicos. Mientras que los riesgos objetivos se repiten con frecuencia por lo que pueden ser estudiados y resulta posible establecer una probabilidad matemática de ocurrencia e intensidad; los específicos son muy poco frecuentes, por lo que la valoración sobre las posibilidades de ocurrencia y consecuencias son subjetivas, siendo 
decisiva la intervención del potencial afectado o de una tercera persona delegada para establecer un criterio sobre su probabilidad y gravedad (Belaunde, 2017).

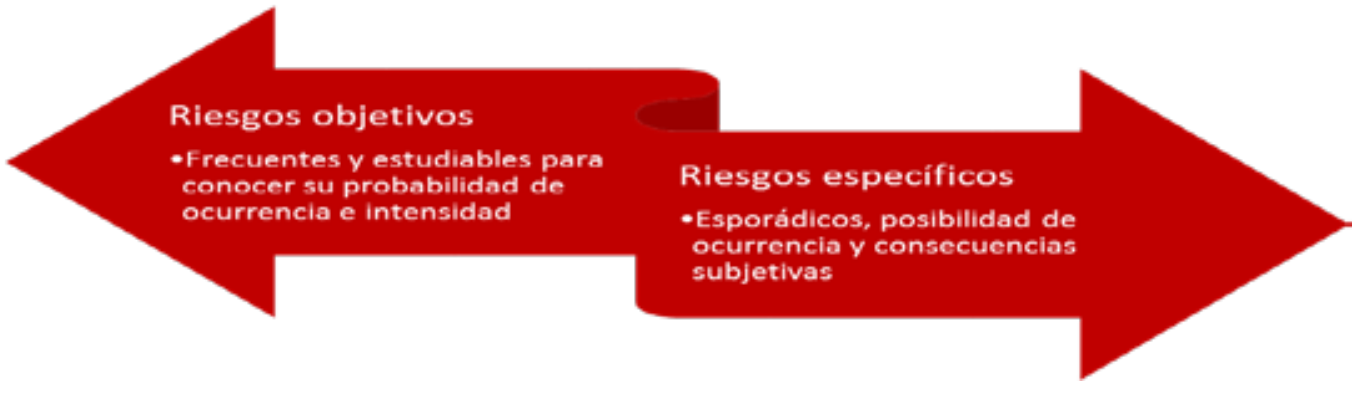

Figura 2. Riesgo objetivo y riesgo especifico

Fuente: elaboración propia

\section{Metodología}

Dentro de la investigación se aplicó un análisis teórico documental, partiendo desde una revisión literaria sobre los diferentes tipos de riesgos empresariales, buenas prácticas administrativas y herramientas para el manejo oportuno de los mismos, complementado con un análisis descriptivo de las mejores metodologías para la disminución o tratamiento del riesgo. Mediante una revisión literaria se visualizará las varíables inmersas en los diferentes tipos de riesgos, impactos y decisiones directivas, que, de no ser oportunas con el tiempo, pueden generar grandes pérdidas económicas en las organizaciones o incluso su cierre por una mala gestión.

Conjuntamente, se aplicó la técnica de investigación teoría fundamentada, misma que parte desde diferentes datos previamente estudiados por revistas y bibliografía previamente citadas en el presente artículo, con la finalidad de definir claramente los diferentes enfoques y respuestas que las áreas directivas de las organizaciones o personal responsable puedan tomar oportunamente.

\section{Resultados}

Los diferentes directivos o personal encargado de tratar los riesgos en las organizaciones utilizan diversas herramientas para tratar, predecir o mitigar los efectos y posibles causas que se puedan generar, ante esto, es necesario tener en mente los recursos tanto físicos, financieros y el personal adecuado para las diversas gestiones o toma oportuna de decisiones.

\subsection{Las actitudes, estrategias y gestión del riesgo}

Para comprender plenamente los riesgos, es necesario delinear sus particularidades, métodos y las actividades sociales que un desastre afectaría específicamente. Desde una perspectiva filosófica se afirma que la verdadera igualdad entre los seres humanos radica en la capacidad de decidir libre y racionalmente una acción que puede adoptar, por ejemplo, ante un determinado riesgo (Bernabé, 2016). 
En este sentido, existen individuos que se desenvuelven por la vida ignorando los riesgos, como si solo existieran para los demás, sin considerar las consecuencias de las diferentes fuentes de riesgos potenciales que le atañen. Cada persona posee un sistema de valoración diferente que le llevará a adoptar distintas actitudes frente a los riesgos que se le presenten, las cuales que se pueden agrupar en seis opciones, además de ignorar el riesgo pueden procurar eliminarlo, asumirlo, reducirlo, transferirlo a terceros o gestionarlo (Bernabé, 2016), tal como se ve evidencia en la Figura 3.

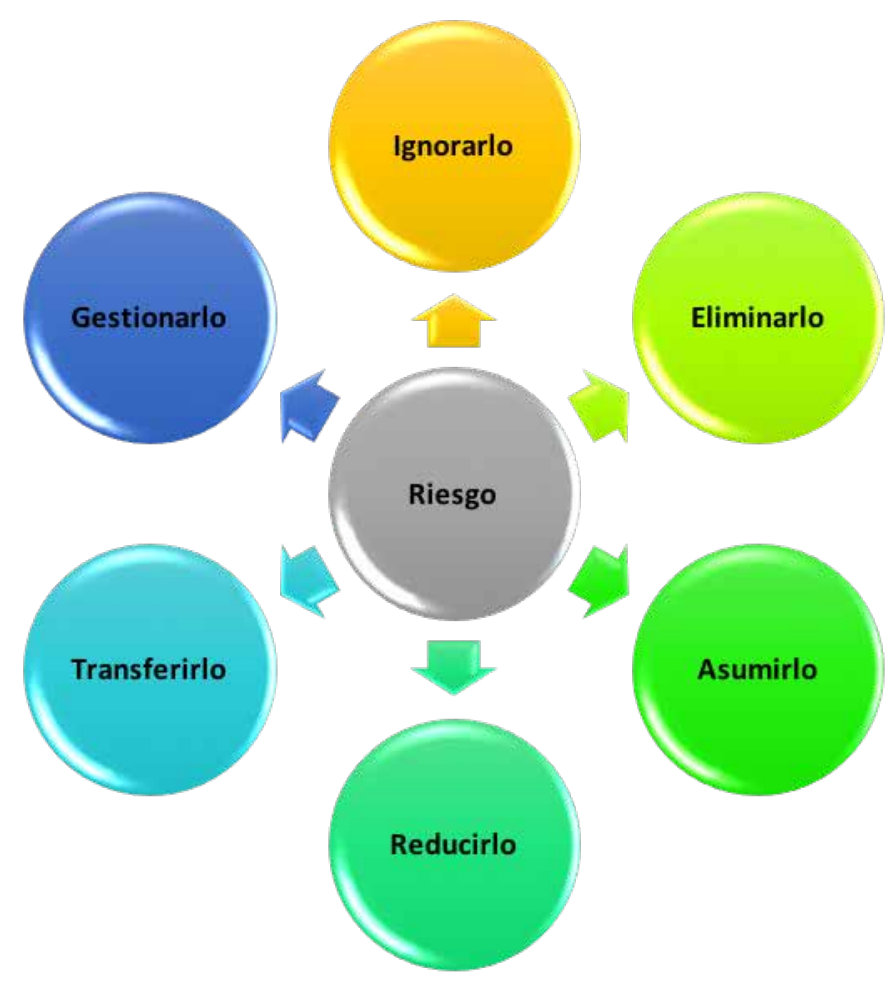

Figura 3. Actitud estratégica frente al riesgo

Fuente: elaboración propia

Ciertos riesgos pueden ser eliminados como cuando se quiere evitar quedar atrapado en un ascensor, en el momento se opta por el uso de las escaleras, ésta es la llamada prevención de los riesgos. Asumir un riesgo implica ser consciente del mismo y a partir de la valoración que se realice puede determinar no realizar acción alguna o crear un fondo paralelo para reponer las eventuales pérdidas que se pudieran presentar. Si el riesgo identificado no resulta posible eliminarlo ni asumirlo, se puede procurar reducirlo, por ejemplo, al diversificar los depósitos de combustible y así protegernos de ese riesgo (Gómez, 2018).

Cuando no es posible adoptar alguna de las opciones anteriores (o no resulta económicamente conveniente hacerlo), se puede prevenir transfiriéndolo a terceros, principalmente utilizando tres instrumentos: empleando un contrato específico, valiéndose de un derivado financiero (opciones de compra, opciones de venta, swaps, etc.) que permitan cubrirse ante la eventualidad, o contratando un seguro específico que provea amparo ante el riesgo identificado (Bernabé, 2016). 
Al decidir gestionar el riesgo implicará identificar fuentes potenciales de riesgo; anticipar la probabilidad de ocurrencia de ciertas situaciones y sus consecuencias a fin de poder implementar acciones para obtener la combinación correspondiente entre el resultado esperado, la variabilidad y libertad de acción, siendo que dicho balance debe estar en función de la capacidad de la empresa para asumirlo (Bernabé, 2016).

\subsection{Pasos para la gestión estratégica del riesgo}

Existen varias metodologías para gestionar los riesgos, las cuales se diferencian mucho si se tratan de una empresa o una persona, riesgos generales o específicos, si existen coberturas naturales o no, etc. A continuación, se propone una metodología integral de diez pasos (Figura 4) para el tratamiento eficiente de la mayoría de los riesgos que puedan presentarse.

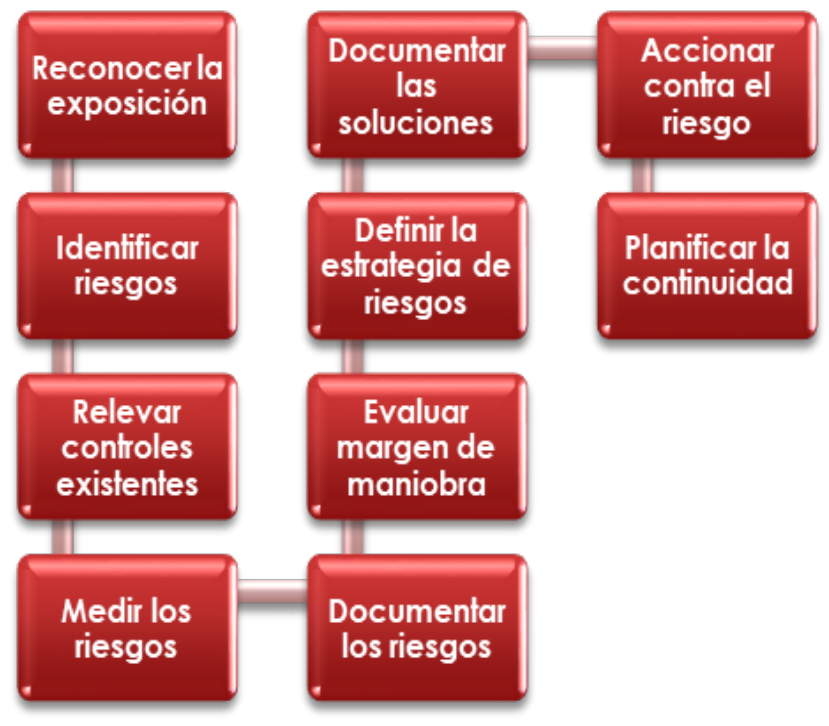

Figura 4. Metodología 10 pasos para la gestión del riesgo

Fuente: elaboración propia

\section{- Reconocer la exposición}

Es fundamental ordenar que las personas tienden a ser vulnerables al riesgo, evaluando sus intereses, patrimonio (seguros, pólizas, etc.) y considerar que los ingresos deben ser protegidos.

\section{- Identificar riesgos}

Es indispensable evaluar el riesgo (pasado, presente y futuro) que puedan presentarse en la organización estableciendo los orígenes, causas y posibles efectos en base a una categoría específica que se detalla a continuación: Comercial o especulativo Vs. Riesgo puro. Particulares Vs. Generales Vs. Catastróficos. Objetivos Vs. Específicos 


\section{- Relevar controles y seguridad existente}

Es necesario analizar los planes de gestión de riesgos anteriores considerando las disposiciones y normativas vigentes por cada país, identificando las seguridades y falencias que las organizaciones públicas y privadas posean con la finalidad de descartar posibles riesgos.

\section{- Medir los riesgos}

Dentro de esta etapa es indispensable establecer las siguientes variables, enfocándose en la realidad empresarial.

1. Calcular la probabilidad matemática de ocurrencia (en el caso que esto sea factible).

2. Estimar la probabilidad subjetiva de frecuencia (en caso de corresponder).

3. Establecer el grado de impacto (o intensidad) de cada riesgo.

4. Determinar la priorización (multiplicando la probabilidad x el impacto).

\section{- Documentar los riesgos}

Es indispensable llevar un registro y control de los diferentes riesgos que se vayan presentando en la organización con la finalidad de tener antecedentes viables para la toma de decisiones, para esto se recomienda generar la matriz de tolerancia a los riesgos y graficar el mapa de riesgos (en el caso de corresponder).

\section{- Evaluar predisposición y margen de maniobra}

Establecer el perfil de riesgo permitirá a la persona, área o responsable establecer un plan de acción en base a:

- Adverso al riesgo

- Neutral al riesgo

- Propenso al riesgo

1. Determinar la capacidad de afrontar los siniestros

2. Identificar los recursos que pueden ser destinados para gestionar o transferir los riesgos 


\section{- Definir la estrategia de riesgos}

1. Decidir el tratamiento de cada riesgo

- Descartar (ignorar / asumir)

- Atender (reducir / eliminar / gestionar)

- Transferir (seguros o derivados financieros)

2. Documentar y asentar (hacer firmar en caso de corresponder)

- Documentar las soluciones

Un registro detallado de las soluciones empleadas ante los posibles riesgos, permitirá tener un antecedente y tomar decisiones con un grado mayor de confiabilidad.

1. Matriz de riesgo residual

2. Matriz de contingencia

- Accionar contra el riesgo

Es indispensable el ejecutar un plan de acción claro, involucrando de forma integran a cada área de la organización con la finalidad de:

1. Asignar los recursos para la gestión de riesgos

2. Implementar el plan de acción desarrollando acciones concretas

3. Documentar lo realizado e informar (en caso de corresponder)

\section{- Planificar la continuidad}

1. Retroalimentación

2. Monitoreo y control

3. Realizar sugerencias y recomendaciones de mejoras

4. Implementar un plan de mejora continua y controles periódicos

\subsection{La necesidad de gestionar los riesgos específicos}

Si se procura gestionar el riesgo se debe tomar en cuenta una de las cuestiones más importantes que es la de considerar el perfil del decidido al analizar un riesgo, dónde lo económicamente 
conveniente es iniciar por discriminar entre los diferentes tipos de stakeholders (amantes o adversos al riesgo), para luego aplicar técnicas y métodos de entrevistas que ayuden a la identificación de riesgos, documentar tanto los riesgos detectados como el perfil de aversión al riesgo para el cuál se elabora el trabajo, medir las probabilidades de ocurrencia y el impacto permitiendo establecer prioridades, planificar las acciones concretas a desarrollar diagramando procesos de respuesta y finalmente implementar dichas actividades incluyendo el control y monitoreo (Córdova, 2017).

La consideración de la subjetividad en la percepción de riesgos se atribuye a Kenneth Arrow, el cuál consciente de la imposibilidad de prever el futuro y las contingencias que éste depara, además de desarrollar una completa teoría de la diversificación basada en la aversión al riesgo de los individuos, este estableció en su teorema de la imposibilidad la inviabilidad del establecimiento de reglas racionales para el logro del bienestar social a partir de funciones de bienestar individuales y a la vez que reconoció la importancia entre la diferenciación de lo que es cuantificable y lo que no.

En este sentido, y al hablar de riesgos específicos, no será posible emplear técnicas cuantitativas como la teoría de la probabilidad, ley de los grandes números, técnicas de muestreo, método de simulación de Montecarlo, la curva en forma de campana y la dispersión en torno a la media, la regresión con observaciones hasta sacar una media, o la teoría de la utilidad (Córdova, 2017).

Si se podrán emplear la confección de perfiles de riesgo, focus group, método Delphi, de consulta a expertos, de análisis de riesgos específicos, comparaciones de a pares, etc. Por regla general, cuanto más específico sea el riesgo; mayor importancia relativa tendrá la experiencia, percepción y propensión (o no) al riesgo que tenga el analista y/o quién decida sobre el tratamiento de un determinado riesgo (Machicao, 2017).

Un correcto programa de gestión de riesgos debe tener un concepto preventivo, debe ser proactivo antes que reactivo. Se debe comenzar con las expectativas y el análisis de riesgos considerándolos desde la fase inicial del proyecto o negocio (Córdova, 2017).

Desde una perspectiva práctica, la gestión de riesgos siempre debe evaluar si los riesgos a los que se enfrenta son simples, complejos o mixtos; y que los riesgos complejos no deben subestimarse y considerarse simples, porque esto solo asegura que se deberá trabajar mucho más en los riesgos de lo que se debería al no darle la relevancia que merece (Machicao, 2017).

Para realizar una correcta gestión del riesgo, debe considerarse no solamente el carácter proactivo, ex-ante de la ocurrencia, sino también la actitud reactiva, ex-post que implica la ejecución del plan de contingencia una vez que ha acontecido el evento previsto (Machicao, 2017).

\subsection{Herramientas para gestionar riesgos}

Entre las diferentes herramientas que permiten diseñar estrategias de mitigación de riesgos empresariales mediante la aplicación de indicadores de cumplimiento, Sulca Córdova, G. C., y Becerra Paguay destacan a la matriz de riesgos como la más adecuada para determinar el plan de 
acción de los procesos que tienen mayor impacto en los riesgos de negocio y ocurren con mayor frecuencia. Mediante estas dos variables, una matriz de riesgos revela los grados de tolerancia a cada riesgo, conformando la herramienta gráfica más importante en la gestión de los riesgos, ya que el empleo de los colores del semafórico, proporciona una rápida visión de las alertas que ameritan atención con urgencia y/o de los temas más importantes a atender (Córdova, 2017).

No obstante, la matriz es una de las varias herramientas que pueden emplearse en una gestión integral de los riesgos. A pesar que el diagrama de Ishikawa se considera una herramienta de gestión de la calidad, de Saeger afirma que no debe limitarse a su objetivo principal y que, a la hora de preparar proyectos, esta herramienta se puede utilizar muy eficientemente para el análisis contextual y en especial para el análisis de riesgos potenciales, o cual es un aspecto que las empresas están considerando cada vez más.

También, los mapas de riesgo se utilizan para ayudarlo a comprender los riesgos en un área o ubicación geográfica específica para que pueda tomar medidas para prevenir o reducir el impacto de posibles eventos. Este tipo de gráfico es especialmente útil cuando es necesario determinar los riesgos que se producen en un determinado espacio físico para que podamos tomar medidas que nos permitan afrontar los riesgos de la forma más eficaz a través de cualquiera de sus seis estrategias. Un ejemplo es observar la distribución de los edificios en una empresa y determinar qué áreas implican menos daño a áreas peligrosas y/o propensas a incendios.

Además, la matriz de contingencia brinda una representación gráfica de los puntos principales del plan de emergencia. No solo puede analizar la frecuencia e impacto de cada riesgo, sino también expresar la prioridad y fuente de cada incidente y las áreas de preocupación de cada persona y las medidas que cada persona responsable debe adoptar (Jiménez Rodríguez, 2016).

\subsection{El desafío para la administración de riesgos}

Aunque la gestión de riesgos específicos reviste una dificultad mayor que los demás riesgos por depender de las percepciones y subjetividades de los intervinientes, es precisamente en este tipo de casos dónde a partir de la individualidad de cada contingencia los especialistas en el análisis de estas eventualidades deben destacarse procurando colaborar en el reconocimiento y evaluación de los riesgos sin interferir en el grado de aversión o propensión que el potencial afectado posea al respecto (Bernstein, 2014).

En este sentido, Brito Gómez afirma que la gestión de riesgos es una nueva tendencia en el mundo empresarial y que, con la aparición del control interno en la década de 1990, con el fin de comprender mejor estos temas, la gestión de riesgos ha jugado un papel de liderazgo en la empresa, aunque en varios países de Latinoamérica, el concepto de riesgo empresarial equivocado es repetitivo, pero debe ser considerado como parte del proceso de aprendizaje (Gómez, 2018).

Al respecto, Barreiro Noa señala que la nueva versión de ISO 9001/2015 tiene como objetivo enfatizar y orientar el enfoque basado en el riesgo e introduce en varias cláusulas que incluyen 
cláusulas de proceso, liderazgo y especialmente planificación, a la vez de considere las amenazas, incertidumbres y riesgos inherentes a cualquier producto, actividades y organizaciones, independientemente de su tamaño y sector económico (Noa, 2017).

Sin embargo, Belaunde advierte que una cultura de gestión de riesgos no está necesariamente relacionada con una gran cantidad de conocimientos técnicos; a veces esto puede hacer que pierda la previsión y se oponga a una buena cultura de gestión de riesgos. Lo importante parece estar más en el tipo de educación recibida y la reflexión cultural de los antepasados. El desarrollo de la cultura de gestión de riesgos aún se encuentra en sus etapas iniciales y si bien aún existen grandes lagunas, el conocimiento técnico sobre la gestión de riesgos en la Latinoamérica no es escaso. A su vez, resalta que la experiencia ha demostrado ampliamente la importancia de las campañas de sensibilización y la formación continua dentro de la organización para desarrollar una cultura de gestión de riesgos y difundir conocimientos resulta clave para fomentar la cultura de la gestión de riesgos, la cual debe realizarse con conceptos vertidos en un lenguaje simple para la comprensión de todos (Belaunde, 2017).

Dentro de las ciencias de la administración existe una deuda con la formación de profesionales de los riesgos, relegando la administración de los mismos a los análisis financieros por un lado y al cálculo de probabilidades que realizan los actuarios por el lado de los seguros. Mientras que más de cien aplicaciones para tablets y smartphones se proponen actualmente para administrar riesgos, abunda la bibliografía y las publicaciones en esta temática; existe una considerable escasez de estudios científicos regionales sobre los numerosos riesgos e implicancias a los que deben enfrentarse los administradores de una organización en Latinoamérica (Sandoval, 2015).

En el mundo empresarial, la gestión de riesgos, ya sea como gerencia interna o como asesoría externa, tiene la necesidad de implantarse con una mayor preponderancia en la estructura de cada entidad ya que, desarrollada adecuadamente, debe ser reconocida como parte de la médula de la organización toda vez que tiene bajo su órbita la no menor responsabilidad de preservar la continuidad del negocio.

Al respecto, Sandoval, A., y Ávila señalan a la gestión de riesgos empresariales (ERM) como un proceso mediante el cual la dirección de una empresa u organización gestiona la amplia gama de riesgos (riesgos operativos y de mercado) que enfrenta el nivel de riesgo que se está dispuesto a asumir en función de los objetivos estratégicos de cada organización (Sandoval, 2015).

En este sentido, en su trabajo sobre el gobierno corporativo, Aristizábal, J., Vásquez, A., y Dávila indagan en la arquitectura de control y la administración del riesgo y afirman que entre las buenas prácticas de gobierno corporativo deben considerarse la administración del riesgo, su evaluación y valoración de los mismos; a la vez que es importante contemplar las actividades de control y traducirlas en políticas y procedimientos que aseguren una adecuada gestión de los riesgos (Aristizábal, 2019).

A su vez, al analizar las nuevas tendencias en la gestión de riesgos del control interno, Barrio Carvajal mantiene que en este entorno de constante cambio global y de las organizaciones en particular, ha aumentado la necesidad de implementar un buen sistema de control interno que 
tenga en cuenta todos los posibles riesgos que puedan ser detectados; debiendo considerarse aspectos como la complejidad, la volatilidad, la ambigüedad y la incertidumbre inherente a cada uno de ellos (Carvajal, 2019).

\subsection{La gestión integral de riesgos}

Los principios de riesgo son un pilar importante para la gestión de control ya que, al alinearse con la estrategia de la empresa, impulsan al cumplimiento de sus objetivos. Las empresas deben mantener una cultura de riesgo fuerte, que les permita a todos los colaboradores estar atentos a los eventos que se pueden presentar y manejar los riesgos de manera responsable (Bautista, 2013).

El compromiso de la alta dirección y de la junta directiva juega un papel muy importante en la gestión integral de los riesgos, con el manejo de la información oportuna podrán tener una visión más holística del futuro de la empresa. De la misma manera la gestión integral debe estar apoyado por procesos de la planificación, evaluación, monitoreo y control, lo que le permitirá tener una gobernanza del riesgo (Bautista, 2013).

La política integral de riesgos establecida por un marco de referencia, objetivos, alcance, definiciones, ciclo de la gestión, funciones y responsabilidad, y su estructura, logrará no tener desviaciones en la buena práctica de la administración de la gestión integral de riesgos, por lo que la transparencia de todos los procesos empresariales será muy decisiva a la toma de decisiones (Bautista, 2013).

\subsection{Buenas prácticas empresarial}

La ética y buenas prácticas empresariales son indispensables a nivel mundial debido a que permiten gestionar de forma clara y oportuna una solución a diferentes problemas de índole económico, financiero, culturales, ambientales, etc., que puedan afectar directa o indirectamente a la organización (EKOS, 2019).

Debido a la naturaleza del riesgo, es indispensable que las organizaciones tanto privadas como públicas deben adoptar medidas para tratar, anticipar o predecir los riesgos, sus causas o posibles efectos, conjuntamente es vital promover un enfoque integral de cada área administrativa, departamentos y encargados con la finalidad de abarcar el riesgo y sus posibles soluciones, mediante herramientas como el teletrabajo o video reuniones se podrá mantener una información constante y clara de cada una de las acciones que se tomen (Morales, 2020).

El manejo correcto de las buenas prácticas empresariales, permite contar con una transparencia ante una posible crisis y problemas que se vayan presentando en las organizaciones por parte de los diferentes riesgos y factores internos o externos de la empresa, permitiendo un manejo multifuncional y accesible en todo momento, situación que se hace más clara en entidades financieras, donde la aplicación de buenas prácticas empresariales son fundamentales para el correcto funcionamiento y credibilidad (Cea, 2018). 


\section{Conclusiones}

El riesgo es una eventualidad cuyos efectos positivos o negativos deben ser tratados de manera específica por cada organización, para ello existen varias metodologías con el fin de prevenir, tratar o evadir sus efectos, la metodología de 10 pasos permite a cada persona, área o encargado una respuesta oportuna y sistemática que orientada con las buenas prácticas empresariales, aportan oportunamente a la toma de decisiones.

La gestión de los riesgos ofrece como ninguna otra actividad dentro del management, el continuo desafío a los administradores de necesariamente combinar las capacidades de ambos hemisferios cerebrales dado que resulta indispensable realizar un exhaustivo análisis racional tanto en la detección de riesgos y de sus de sus implicancias, como a la vez el desarrollar soluciones creativas para atender esas eventualidades.

Mientras exista un futuro incierto, este siempre nos proporcionará nuevos retos y la oportunidad de superarlos con éxito o sumirnos en un profundo fracaso, dependiendo de la atención, capacidad y habilidad de quién deba administrar. Por eso, con ética y responsabilidad, los administradores estamos obligados a asumir con profesionalismo nuestro rol y preservar a las organizaciones ( $y$ las fuentes de trabajo que de ella dependen), de todos los riesgos que nuestra pericia nos permita gestionar.

Las buenas prácticas empresariales brindan un sistema claro de confiabilidad para las empresas, estado o sociedad debido a que aportan con herramientas útiles para medir los riesgos, mediante un tablero de control que permite tener los indicadores relevantes para la toma de decisiones.

El establecimiento de metodologías, reglas y procedimientos para los análisis y evaluación de los riesgos que tiene la empresa, logra evitar sus pérdidas o gastos, generando más valor a la empresa y atractiva para los inversionistas.

Contar con una alta dirección capaz de involucrar de manera integral cada área de la organización, enfatizando su importancia ante los planes o tratamientos de los riesgos permite tener una visión más holística del futuro de la empresa, apoyando los procesos, planificación, monitoreo y control de la gobernanza del riesgo. 


\section{Referencias}

Aristizábal, J. V. (2019). La arquitectura de control y la.

Bautista, R. (2013). Cada vez piden más explicaciones sobre la gestión de riesgos. Gerencia de riesgos y seguros España, 7-15.

Belaunde, G. (2017). La Importancia de la Cultura de Gestión de Riesgos. Obtenido de https://gestion.pe/blog/ riesgosfinancieros/2017/05/la-importancia-de-la-cultura-de-gestion-de-riesgos.html/

Bernabé, M. A. (2016). Gestión de Riesgo en el Ecuador. Quito: Editorial ESPE.

Bernstein, P. L. (2014). Against the gods. The remarkable story of risk. Hardcover. USA., 1-5.

Carvajal, B. (2019). Nuevas tendencias en la gestión de riesgos del Riesgo. Colombia: Auditoría pública: revista de los Órganos Autónomos de Control. Obtenido de https://dialnet.unirioja.es/servlet/articulo?codigo $=6966918$

Cea, C. B. (2018). Nuevas fronteras de la Responsabilidad Social de la Empresa. Revista de Responsabilidad Social de la Empresa, 1-30. Obtenido de https://www.accioncontraelhambre.org/sites/default/files/documents/revista_rse_n29_5jul.pdf\#page=17

Córdova, S. (2017). Control interno Matriz de riesgo: Aplicación metodología COSO II. Revista Publicando, 4-12. Obtenido de https://revistapublicando.org/revista/index.php/crv/article/view/686

EKOS. (2019). Buenas prácticas empresariales. Revista Ekos, 1-5. Obtenido de https://www.ekosnegocios.com/ articulo/buenas-practicas-empresariales

Gómez, B. (2018). El riesgo empresarial. 1-5. Obtenido de http://scielo.sld.cu/scielo.php?script=sci_arttext\&amp;pid=S2218-

Jiménez Rodríguez, E. J. (2016). El riesgo operacional. Metodología para su medición y control. Delta Publicaciones., 7.

Machicao, J. (2017). La diferencia entre riesgos complejos y riesgos simples. GestioDinámica., 5-9. Obtenido de https://www.academia.edu/32123032/Riesgos_simples_y_riesgos_complejos

Morales, D. (Junio de 2020). Teletrabajo como estrategia de competitividad y desarrollo para las empresas del Ecuador. ERUDITUS, 1-12. https://doi.org/10.35290/re.v1n2.2020.318

Noa, B. (2017). Administración financiera del riesgo empresarial. Editorial Académica Universitaria. Obtenido de http://localhost/xmlui/handle/123456789/5 
Sandoval, A. y. (2015). Administración de riesgos. Aplicación de la herramienta integrada de control interno y administración de riesgos, enmarcada en buen gobierno corporativo para pequeñas y medianas empresas en Colombia. Universidad del Externado, 35-85. https://doi.org/10.2307/j.ctv13vddx6.6 